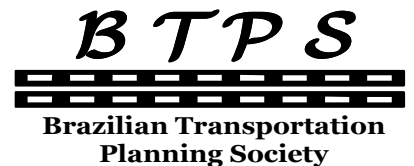

Planning Society
Journal of Transport Literature

Vol. 8, n. 3, pp. 270-289, Jul. 2014

Research Directory
JTL | RELIT

www.transport-literature.org ISSN 2238-1031

\title{
Aprimoramento do desempenho ambiental de polos geradores de viagem (PGV carga): estudo de caso do terminal Sepetiba Tecon
}

[Improving the environmental performance of trip generators: case study Sepetiba Tecon terminal]

\author{
Cintia Machado de Oliveira, Amanda Fernandes Ferreira, Márcio de Almeida D’Agosto, \\ Ilton Curty Leal Junior, Vanessa de Almeida Guimarães*
}

Federal University of Rio de Janeiro - Brazil, Fed. University of Rio de Janeiro - Brazil, Federal University of Rio de Janeiro - Brazil, Fluminense Federal University - Brazil, Federal University of Rio de Janeiro - Brazil

Submitted 6 May 2013; received in revised form 30 Sep 2013; accepted 1 Nov 2013

\begin{abstract}
Resumo
Este artigo objetiva identificar como medidas que promovem a sustentabilidade no transporte de carga podem afetar o desempenho ambiental de um PGV Carga. Para tanto o método proposto por Guimarães e Leal Jr (2013) foi aplicado na avaliação do Terminal Sepetiba Tecon, que faz parte do Porto de Itaguaí. Aspectos econômicos e ambientais foram avaliados considerandose alguns atributos, a citar, valor de serviço que se refere ao serviço produzido e influência ambiental que se refere ao consumo de energia, emissão de gases de efeito estufa, poluição atmosférica, poluição da água e do solo, consumo de água e consumo de material. Foram considerados veículos rodoviários e equipamentos de movimentação de carga para estimar a emissão de Gases de Efeito Estufa (GEE) e poluentes atmosféricos ligados à atividade portuária. Foi realizada ainda uma análise de sensibilidade. Está análise teve como intuito avaliar as variações que podem ocorrer em função da diferença nos pesos atribuídos a cada indicador utilizado na análise e verificar como esta variação altera o resultado final
\end{abstract}

Palavras-Chave: polos geradores de viagens de carga, desempenho ambiental, aplicação de método.

\begin{abstract}
This article aims to identify how measures that promote sustainability in freight transportation can affect the environmental performance of a load trip generator. To achieve this goal the method proposed by Guimarães and Leal Jr (2013) was applied in the evaluation of Sepetiba Tecon Terminal, which is part of the Port of Itaguai. Economic and environmental aspects were evaluated considering some attributes, such as value of service that refers to the service produced and environmental influence that refers to energy consumption, emission of greenhouse gases, air pollution, water pollution and ground water consumption and material consumption. Were considered road vehicles and cargo handling equipment to estimate the emission of Greenhouse Gases (GHG) and air pollutants related to port activity. It was also performed a sensitivity analysis. This analysis had the intention to assess changes that may occur due to the difference in the weights assigned to each indicator used in the analysis and see how this variation changes the outcome.
\end{abstract}

Key words: load trip generators, environmental performance, application of method.

*Email: vanessaaguim@gmail.com.

\section{Recommended Citation}

Oliveira, C. M., Ferreira, A. F., Dagosto, M. A., Leal Jr. I. C. and Guimarães, V. A. (2014) Aprimoramento do desempenho ambiental de polos geradores de viagem (PGV carga): estudo de caso do terminal Sepetiba Tecon. Journal of Transport Literature, vol. 8, n. 3, pp. 270-289.

- JTL/RELIT is a fully electronic, peer-reviewed, open access, international journal focused on emerging transport markets and published by BPTS - Brazilian Transport Planning Society. Website www.transport-literature.org. ISSN 2238-1031. 


\section{Introdução}

O conceito de um polo gerador de viagens pode ser entendido como um local com potencial de gerar um significativo número de viagem, exigindo uma infraestrutura que comporte locais de embarque, desembarque, estacionamento e também como um local capaz de gerar impactos significativos em relação a aspectos socioambientais e econômico-financeiros. (CET, 1983; DENATRAN, 2001; Portugal e Goldner, 2003; Tolfo e Portugal, 2006; Kneib et al., 2010; Souza et al. 2010; Rede PGV, 2013).

Para o Denatran (2001) e CET (1983), devido à capacidade de atração de um grande numero de viagens, um PGV causa reflexos negativos na circulação do entorno viário, prejudicando, em alguns casos, a acessibilidade à região.

Com o intuito de evitar impactos negativos no entorno de um PGV, um planejamento adequado de um terminal de carga pode colaborar com identificação de restrições operacionais que influenciam os índices de desempenho e produtividade, além de identificar os impactos ambientais causados pela operação de transportes e auxiliar na promoção de melhorias.

Este artigo tem como objetivo identificar como medidas que promovem a sustentabilidade no transporte de carga podem afetar o desempenho ambiental de um PGV Carga. Para isso, adota o método de avaliação do desempenho ambiental de PGVs carga que possibilita conciliar o aspecto econômico e ambiental.

Os dados analisados correspondem ao período 2011 e serão considerados apenas os impactos provenientes da operação de transporte. Além disso, serão considerados apenas os impactos das atividades dentro do terminal, não levando em conta a influência destes em seu entorno. O estudo será realizado no Porto de Itaguaí, situado na cidade de Itaguaí, Rio de Janeiro e analisará o terminal de contêineres Sepetiba Tecon. 
A partir desta introdução, este trabalho se divide em 4 seções. Os conceitos relacionados ao método de avaliação do desempenho ambiental de PGVs carga e os procedimentos metodológicos são sucintamente apresentados na Seção 1, sendo a descrição e a aplicação ao caso em estudo e os resultados encontrados apresentados na Seção 2. Na Seção 3 é apresentada a aplicação do método de avaliação do desempenho ambiental de PGV's carga. Finalmente, na última seção encontram-se as conclusões, limitações e sugestões de aprimoramento deste trabalho.

\section{Procedimentos Metodológicos}

Este trabalho teve como base uma pesquisa documental e bibliográfica que permitiram conhecer e dominar uma seleção de métodos de avaliação de desempenho, sua estrutura e variáveis empregadas para a mensuração do desempenho de todos os portos analisados.

Para realização da avaliação de desempenho ambiental dos portos, utilizou-se o método apresentado na figura 1, que é o método de avaliação do desempenho ambiental de PGVs carga desenvolvido por Guimarães et al (2013). O método consiste em 8 passos, sendo seis deles divididos em níveis como parte de um método dinâmico de avaliação de desempenho para a elaboração de uma proposta de melhorias a ser empregada em um PGV carga. As informações de sua aplicação ao Porto de Itaguaí serão apresentadas na próxima seção.

Conforme previsto no passo 6 , nível 1 , do método apresentado na figura 1 , é preciso fazer o cálculo das medidas para se determinar o desempenho padrão dos portos analisados e assim, poder realizar as etapas seguintes. Para tanto, foram aplicadas as equações de 1 a 6 .

Foram considerados veículos rodoviários e equipamentos de movimentação de carga para estimar a emissão de Gases de Efeito Estufa (GEE) e poluentes atmosféricos ligados à atividade portuária. Para o cálculo das emissões de GEEs, que utilizou como referência a metodologia do IEAVAERJ (2011) e, para os poluentes atmosféricos, aplicou a metodologia aplicada no porto de Long Beach (LLC, 2012).

As emissões totais de poluentes e GEE, foram calculadas aplicando-se as equações 2 e 3 , respectivamente. 


$$
\begin{gathered}
E T p=\sum_{j=1}^{n}\left\{\left[\left(\mathrm{p}_{\mathrm{j}} \times \mathrm{ho}_{\mathrm{j}} \times \mathrm{fc}_{\mathrm{j}} \times \mathrm{fep}_{\mathrm{j}} \times \mathrm{fcc}_{\mathrm{j}} \times \mathrm{fct}_{\mathrm{j}}\right) \times \mathrm{ne}_{\mathrm{j}}\right]+\sum_{j=1}^{n}[(f e p v \times d) \times q v]\right\} \times \lambda \\
E T g=\sum_{j=1}^{n}\left[\left(p_{j} \times h o_{j} \times f c_{j} \times f e g_{j} \times f c c_{j} \times f c t_{j}\right) \times n e_{j}\right]+\sum_{j=1}^{n}\{[(d / c) \times f e g v] \times q v\}
\end{gathered}
$$

Onde: ETp: emissões totais de poluentes atmosféricos (t/ano)

ETg: emissões totais de gases de efeito estufa (t/ano)

p: potência nominal do motor do equipamento de movimentação $(\mathrm{hp})$

ho: horas de operação anual do equipamento de movimentação (h/ano)

fc: fator de carga dos equipamentos de movimentação (carga média usada durante as operações normais em comparação com plena carga)

fep: fator de emissão de poluentes dos equipamentos de movimentação (t/hph)

feg: fator de emissão de GGE dos equipamentos de movimentação (t/hph)

fcc: fator de correção de combustível para refletir as mudanças nas propriedades dos combustíveis que têm ocorreu ao longo do tempo

fct: fator de controle para refletir as mudanças nas emissões devido à instalação de tecnologias de redução de emissão não originalmente refletida na emissão fatores

ne: número de equipamentos de movimentação por tipo

fepv: fator de emissão de poluentes dos equipamentos rodoviários $(\mathrm{t} / \mathrm{km})$

fegv: fator de emissão de GGE dos equipamentos rodoviários (t/l)

d: distância percorrida (km/ano)

c: consumo de combustível $(\mathrm{km} / \mathrm{l})$ 
qv: quantidade de veículos rodoviários ${ }^{1}$

$\lambda=$ fator de conversão para tonelada equivalente de $\mathrm{CO} 2$

Para o calculo do consumo de água utilizou-se a Equação 3.

$$
C T a=q f \times c a \times d
$$

Onde: CTa: consumo total de água (1/ano)

qf: quantidade de funcionários

ca: consumo médio diário de água por funcionário (litros)

d: dias trabalhados em um ano

Para o cálculo da poluição da água e do solo aplicou-se a Equação 4. Considerou-se apenas a emissão de esgoto.

$$
C T a=q f \times c a \times d
$$

qf: quantidade de funcionários

qe: média diária de emissão de esgoto, por pessoa (1/dia)

d: dias trabalhados em um ano

Para o cálculo do consumo de materiais utilizou-se a Equação 5.

$$
C M=q f \times m t
$$

Onde: CM: consumo de materiais $(\mathrm{t})$

qf: quantidade de funcionários

mt: média anual do consumo de materiais por funcionário em um terminal portuário (t/ano)

\footnotetext{
${ }^{1}$ A quantidade de veículos foi obtida através de dados disponíveis em Guimarães e Leal Jr (2013)
} 
Para o cálculo do consumo de energia, aplicou-se a Equação 6.

$$
C t e=[(d / c) \times k \times q v]+\sum_{j=1}^{n}(p \times h o \times 3,6) \times n e+\sum_{j=1}^{n}(k \times c \times h o) \times n e
$$

Onde: CTe: Consumo total de energia (MJ)

d: distância (km/ano)

c: consumo de combustível (em $\mathrm{km} / \mathrm{l}$ para veículos rodoviários e em $1 / \mathrm{h}$ para equipamentos de movimentação)

$\mathrm{k}$ : fator de energia em função do combustível usado (MJ/l)

qv: quantidade de veículos rodoviários

p: potência do motor elétrico do equipamento de movimentação (sendo $1 \mathrm{kwh}=3,6$

MJ)

ho: horas de operação anual (h/ano)

ne: número de equipamentos de movimentação

Ainda com base em Guimarães e Leal Jr (2013), o consumo total de energia, foi subdividido em renovável e não renovável. Para energia renovável, considerou-se o consumo dos equipamentos elétricos, tendo em vista que, no Brasil, utilizam-se fontes hidroelétricas, assim como 4,7\% do consumo dos equipamentos e veículos a diesel, Para os demais, considerou-se como não-renovável. Essa consideração foi fundamentada no fato do diesel brasileiro possuir $5 \%$ de mistura de biodisel assumindo assim, que o poder calorífico do biodiesel é $4,7 \%$ menor que o do óleo diesel em um litro.

Em seguida, atendendo ao passo 6, nível 2, adotou-se a normalização das medidas, conforme Equação 7:

$$
M N_{i j}=\frac{M_{i j \min }}{M_{i j}} \text { para j: } 1 . .10
$$


Onde: $\mathrm{MN}_{\mathrm{ij}}-$ Resultado normalizado da medida $\mathrm{j}$ para o porto $\mathrm{i}$;

$$
\begin{aligned}
& \mathrm{M}_{\mathrm{ijmin}} \text { - Menor valor da medida j para o porto } \mathrm{i} \text {; } \\
& \mathrm{M}_{\mathrm{ij}} \text { - Valor da medida } \mathrm{j} \text { para o porto } \mathrm{i} \text {. }
\end{aligned}
$$

Para atender ao passo 7, nível 1, que trata da determinação do desempenho global, adotou-se a Equação 8 para que fosse possível a agregação das medidas.

$$
M N A_{i}=\sum_{j=1}^{m} P_{j} M N_{i j} \text { para i: } 1 . .4
$$

Onde: $\mathrm{MNA}_{\mathrm{ij}}$ - Resultado agregado das medidas normalizadas j para o porto $\mathrm{i}$;

$$
\mathrm{P}_{\mathrm{j}}-\text { Peso atribuído à medida } \mathrm{j} \text {; }
$$

$\mathrm{MN}_{\mathrm{ij}}$ - Resultado normalizado da medida j para o porto $\mathrm{i}$.

Atendendo ao passo 8, nível 1, para realização do teste de sensibilidade adotou-se a Equação 9:

$$
M N A S_{i j}=P A \sum_{j=1}^{k} M N A_{i j}+(1-P A) \sum_{j=k+1}^{m} M N A_{i j} \text { para i: } 1 . .4, \mathrm{k}=4, \mathrm{~m}=10
$$

Onde: $\mathrm{MNAS}_{\mathrm{ij}}$ - Resultado da análise de sensibilidade sobre o valor agregado das medidas normalizadas j para o porto i;

PA - Peso atribuído ao aspecto socioambiental (PA: 1 .. 0);

$\mathrm{MNA}_{\mathrm{ij}}$ - Resultado agregado das medidas normalizadas j para o porto i. 


\section{Aplicação do método de avaliação do desempenho ambiental de PGV's carga}

O método consiste na aplicação de 8 passos os quais estão descritos nas seções 3.1 à 3.8. A partir dele foi realizado uma avaliação de desempenho e a elaboração de um proposta de melhoria para um PGV carga.

\subsection{Passo 1 - Definições iniciais}

No passo inicial identificam-se os tipos de PGV carga e suas características. O Porto de Itaguaí fica localizado na cidade de Itaguaí, Rio de Janeiro e possui uma área total de 7,4 milhões de $\mathrm{m}^{2}$. É administrado pela Companhia Docas do Rio de Janeiro (CDRJ) e possui 4 terminais, a citar, TEMIN - CPBS (minério), THYRSSEN KRUPP - CSA (usina siderúrgica), TECAR - CSN (minério e carvão) e Sepetiba TECON (contêineres). Em 2011 o porto movimentou 58,1 milhões de toneladas, sendo 55,4 milhões de toneladas de granéis sólidos e 2,7 milhões de toneladas de carga geral, o que representa 18,8\% do total de carga movimentada através de portos no Brasil (CNT 2012).

Para a realização desse estudo foi analisado o terminal Sepetiba TECON que faz movimentação de contêineres. Os aspectos considerados para realização dessa avaliação foram valor do serviço e influência ambiental. O Terminal Sepetiba Tecon é administrado pela Companhia Siderúrgica Nacional (CSN) sendo o maior terminal de contêineres do Rio de Janeiro, chegando a movimentar 300 mil TEUs por ano. Além disso, esse terminal chega a movimentar 1 milhão de toneladas de produtos siderúrgicos por ano (CSN, 2008). O terminal de Sepetiba TECON possui 3 berços e uma área total de $400.000 \mathrm{~m}^{2}$, sendo a extensão atual do cais de 810 metros, do calado profundidade do canal de acesso 20 metros, profundidade do berço de atração 14,3 metros e uma área de armazenagem de $368.153 \mathrm{~m}^{2}$. Este terminal movimentou em 2012 a quantia de 216.460 contêineres (ABRATEC, 2013). A relação dos equipamentos utilizados na operação do terminal pode ser vista na Tabela 1. 


\section{Tabela 1 - Quantitativo de equipamentos do Terminal Sepetiba TECON}

\begin{tabular}{|c|c|c|c|c|c|c|c|c|}
\hline \multirow[b]{2}{*}{ Porto } & \multirow[b]{2}{*}{$\begin{array}{l}\text { Movimentação } \\
\text { (TEU's) }\end{array}$} & \multicolumn{7}{|c|}{ Quantidade de Equipamentos } \\
\hline & & Portêiner & $M H C$ & $\begin{array}{l}\text { Caminhão } \\
\text { Trator }\end{array}$ & Semi reboque & Reachstacker & Empilhadeira & $R T G$ \\
\hline $\begin{array}{l}\text { Sepetiba } \\
\text { TECON }\end{array}$ & 225.255 & 4 & 2 & 17 & 12 & 14 & 27 & 2 \\
\hline
\end{tabular}

O artigo abrange apenas parte do sistema, já que não considera todas as alternativas de transporte e a rede completa. O nível de análise é o estratégico, pois buscou-se definir a rede logística mais adequada. O ponto de vista da análise do desempenho é o do operador logístico, responsável pelo transporte do material e prestador de serviço da Vale.

\subsection{Passo 2 - Atributos e Pesos}

O segundo passo consiste na escolha e na ponderação dos atributos relacionados aos aspectos valor de serviço e influência ambiental, e dos pesos para cada um dos atributos escolhidos. Com base em Guimarães e Leal Jr (2013), optou-se por considerar para o aspecto valor de serviço: serviço produzido e quanto ao aspecto influência ambiental, optou-se por considerar: consumo de energia, emissão de gases de efeito estufa, poluição atmosférica, poluição da água e do solo, consumo de água e consumo de material.

Para determinação dos pesos aplicados a cada atributo relacionado aos aspectos, valor de serviço e influência ambiental, como não se teve parâmetro de avaliação, adotou se uma divisão igualitária, assim, cada atributo relacionado ao aspecto influência ambiental recebeu igual fração de $16,67 \%$ de peso, já para o aspecto valor de serviço, como só tinha um atributo, este, recebeu um peso de $100 \%$. Os resultados são apresentados na Tabela 2.

Com intuito de avaliar as variações que podem ocorrer em função da diferença nos pesos atribuídos a cada indicador, foi realizada uma análise de sensibilidade. Esta análise se baseia na atribuição de diferentes pesos para os atributos de forma a se verificar como esta variação altera o resultado final. Os resultados dessa análise se encontram no item 3 que trata da "Análise dos resultados e análise de sensibilidade". 


\begin{tabular}{|c|c|c|c|c|c|c|}
\hline \multicolumn{2}{|c|}{$\begin{array}{c}\text { PASSO } 1 \\
\text { DEFINIÇÕES INICIAIS }\end{array}$} & \multicolumn{2}{|c|}{$\begin{array}{c}\text { PASSO } 2 \\
\text { ATRIBUTOS E PESOS }\end{array}$} & \multicolumn{2}{|c|}{$\begin{array}{c}\text { PASSO } 3 \\
\text { INDICADORES }\end{array}$} & $\begin{array}{c}\text { PASSO } 4 \\
\text { DESEMPENHOPADRÃO }\end{array}$ \\
\hline $\begin{array}{c}\text { Nível1 } \\
\text { PGV carga }\end{array}$ & $\begin{array}{l}\text { - Tipo PGV carga e suas } \\
\text { características }\end{array}$ & \multirow{2}{*}{$\begin{array}{c}\text { Nível } 1 \\
\text { Pesquisa }\end{array}$} & \multirow{2}{*}{$\begin{array}{l}\text { - Revisão bibliográfica e } \\
\text { documental a fim de se } \\
\text { identificar os atributos a } \\
\text { serem considerados no } \\
\text { estudo }\end{array}$} & \multirow[b]{2}{*}{$\begin{array}{l}\text { Nível1 } \\
\text { Pequisa }\end{array}$} & \multirow{2}{*}{$\begin{array}{c}\text { - Pesquisa bibliográfica e } \\
\text { documental e/ou de } \\
\text { campo para } \\
\text { levantamento dos } \\
\text { possíveis indicadores } \\
\text { associados a cada } \\
\text { atributo } \\
\end{array}$} & \multirow[b]{3}{*}{$\begin{array}{c}\text { - Estudo do nível de desempenho dos } \\
\text { benchmarks (externo ou interno) dos } \\
\text { terminais intermodais de carga em cada } \\
\text { medida }\end{array}$} \\
\hline $\begin{array}{l}\text { Nível2 } \\
\text { Terminal }\end{array}$ & $\begin{array}{l}\text {-Seleção e caracterização } \\
\text { do terminal } \\
\text { - Operaçōes e suas } \\
\text { características } \\
\text {-Impactos ambientais }\end{array}$ & & & & & \\
\hline $\begin{array}{l}\text { Nível 3 } \\
\text { Desempenho } \\
\text { Ambiental }\end{array}$ & $\begin{array}{l}\text { - Categorias e aspectos } \\
\text { para avaliação; } \\
\text { - Ponto de vista da } \\
\text { avaliação; } \\
\text { - Nível da análise; } \\
\text {-Escopo da_avaliação. }\end{array}$ & $\begin{array}{l}\text { Nível2 } \\
\text { Escolha e } \\
\text { ponderação }\end{array}$ & $\begin{array}{l}\text { - Seleção, priorização e } \\
\text { ponderação dos } \\
\text { atributos }\end{array}$ & $\begin{array}{l}\text { Nível2 } \\
\text { Escolha }\end{array}$ & $\begin{array}{c}\text { - Determinação dos } \\
\text { indicadores baseados } \\
\text { nos atributos escolhidos } \\
\text { por meio da pesquisa } \\
\text { com especialistas }\end{array}$ & \\
\hline \multicolumn{2}{|c|}{$\begin{array}{l}\text { SAÍDA } \\
\text { Descrição básica dos parâmetros que } \\
\text { orientam a avaliação de desempenho } \\
\text { ambiental em PGVs carga }\end{array}$} & \multicolumn{2}{|c|}{$\begin{array}{l}\text { SAíDA } \\
\text { Lista dos aspectos, atributos e seus } \\
\text { respectivos pesos }\end{array}$} & \multicolumn{2}{|c|}{$\begin{array}{l}\text { SAÍDA } \\
\text { Tabela contendo aspectos, atributose } \\
\text { indicadores a ser em avaliados }\end{array}$} & $\begin{array}{l}\text { SAÍDA } \\
\text { Tabela ou lista com o nível de } \\
\text { desempenho dos benchmarks em cada }\end{array}$ \\
\hline \multicolumn{2}{|c|}{$\begin{array}{l}\text { PASSO } 8 \\
\text { MELHORIAS }\end{array}$} & \multicolumn{2}{|c|}{$\begin{array}{l}\text { PASSO } 7 \\
\text { AVALIAÇÃO }\end{array}$} & \multicolumn{2}{|c|}{$\begin{array}{c}\text { PASSO } 6 \\
\text { PROCESSAMENTO }\end{array}$} & $\begin{array}{c}\text { PASSO } 5 \\
\text { COLETADE DADOS }\end{array}$ \\
\hline \multirow{2}{*}{$\begin{array}{c}\text { Nível } 1 \\
\text { Sensibilidade }\end{array}$} & \multirow{2}{*}{$\begin{array}{l}\text { - Análise de } \\
\text { sensibilidade }\end{array}$} & $\begin{array}{c}\text { Nível } 1 \\
\text { Determinação }\end{array}$ & $\begin{array}{l}\text { - Determinação do nível } \\
\text { de desempenho global }\end{array}$ & $\begin{array}{l}\text { Nível } 1 \\
\text { Medidas }\end{array}$ & - Cálculo das medidas & \multirow{3}{*}{$\begin{array}{l}\text { - Pesquisa bibliográfica e documental e/ou } \\
\text { de campo para determinar os valores dos } \\
\text { indicadores }\end{array}$} \\
\hline & & $\begin{array}{l}\text { Nível } 2 \\
\text { Análise }\end{array}$ & $\begin{array}{l}\text { - Análise do } \\
\text { desempenho global } \\
\text { do(s) terminal(is) }\end{array}$ & $\begin{array}{l}\text { Nível } 2 \\
\text { Método }\end{array}$ & $\begin{array}{l}\text { - Seleção do método de } \\
\text { agregação a serusado } \\
\text { para avaliação dos } \\
\text { terminais }\end{array}$ & \\
\hline $\begin{array}{c}\text { Nível } 2 \\
\text { Aprimoramento }\end{array}$ & $\begin{array}{l}\text { - Proposta de melhorias } \\
\text { - Criação de cenários } \\
\text { (implementação teórica } \\
\text { das melhorias) }\end{array}$ & $\begin{array}{c}\text { Nível } 3 \\
\text { Comparação }\end{array}$ & $\begin{array}{l}\text { - Comparação com os } \\
\text { níveis do(s) } \\
\text { benchmark(s) }\end{array}$ & $\begin{array}{c}\text { Nível3 } \\
\text { Aplicação }\end{array}$ & $\begin{array}{l}\text { - Aplicação do método } \\
\text { escolhido }\end{array}$ & \\
\hline \multicolumn{2}{|c|}{$\begin{array}{l}\text { SAÍDA } \\
\text { Novo resultado da avaliação dos terminais } \\
\text { com base no seu desempenho ambiental }\end{array}$} & \multicolumn{2}{|c|}{$\begin{array}{l}\text { SAí́A } \\
\text { Resultado da avaliação dos terminais com } \\
\text { base no seu desempenho ambiental }\end{array}$} & \multicolumn{2}{|c|}{$\begin{array}{l}\text { SAíDA } \\
\text { Resultado da aplicação do método } \\
\text { selecionado }\end{array}$} & $\begin{array}{c}\text { SAÍDA } \\
\text { Tabela com os valores dos indicadores }\end{array}$ \\
\hline
\end{tabular}

\section{Figura 1- Método de avaliação do desempenho ambiental de PGVs carga ${ }^{2}$}

\footnotetext{
${ }^{2}$ Fonte: Leal Jr (2010); Leal Jr e D’Agosto (2010); Souza et al. (2010); Leal Jr e D’Agosto (2011); LLC (2011a, 2011b e 2012); D’Agosto et al. (2012).
} 
Tabela 2 - Atributos considerados para a avaliação e seus respectivos pesos ${ }^{3}$ Valor do Serviço Influência Ambiental

\begin{tabular}{lclc}
\hline Atributos & Peso & Atributos & Peso \\
\hline \multirow{3}{*}{ Serviço Produzido } & & Consumo de Energia & $16,67 \%$ \\
& \multirow{2}{*}{$100,00 \%$} & Emissão de Gases de Efeito Estufa & $16,67 \%$ \\
& & Poluição Atmosférica & $16,67 \%$ \\
& & Poluição da Água e do Solo & $16,67 \%$ \\
& & Consumo de Água & $16,67 \%$ \\
& & Consumo de Material & $16,67 \%$ \\
\hline
\end{tabular}

\subsection{Passo 3 - Indicadores}

O terceiro passo consiste na seleção dos indicadores e medidas associadas com os atributos. Para isso, um conjunto de indicadores associados a cada atributo escolhido previamente é determinado e combinado para formar uma única medida de desempenho para cada um dos atributos (Tabela 3). Para seleção desses indicadores, tomou-se como base Guimarães e Leal $\operatorname{Jr}(2013)$.

Tabela 3 - Atributos de avaliação, indicadores e medidas e desempenho dos portos ${ }^{4}$

\begin{tabular}{|c|c|c|c|c|c|c|c|}
\hline \multirow{2}{*}{$\begin{array}{l}\text { Aspectos } \\
\text { Atributos }\end{array}$} & \multirow{2}{*}{$\begin{array}{c}\begin{array}{c}\text { Valor de } \\
\text { Serviço }\end{array} \\
\begin{array}{c}\text { Serviço } \\
\text { Produzido }\end{array}\end{array}$} & \multicolumn{6}{|c|}{ Influência Ambiental } \\
\hline & & Consumo & de Energia & $\begin{array}{c}\text { Poluição } \\
\text { da Agua } \\
\text { do Solo }\end{array}$ & $\begin{array}{l}\text { Gases } \\
\text { Efeito } \\
\text { Estufa }\end{array}$ & $\begin{array}{c}\text { Poluição } \\
\text { Atmosférica }\end{array}$ & $\begin{array}{c}\text { Consumo de } \\
\text { água }\end{array}$ \\
\hline Indicadores & $\begin{array}{l}\text { Numero de } \\
\text { Contêineres } \\
\text { Movimentad } \\
\text { os }\end{array}$ & $\begin{array}{c}\text { Energia } \\
\text { Total }\end{array}$ & $\begin{array}{c}\text { Energia } \\
\text { Não } \\
\text { Renovável }\end{array}$ & $\begin{array}{c}\text { Emissão } \\
\text { de Esgoto }\end{array}$ & $\begin{array}{c}\text { Total de } \\
\text { emissões } \\
\left(\mathrm{CO}_{2}\right. \\
+\mathrm{CH}_{4}+ \\
\left.\mathrm{N}_{2} \mathrm{O}\right) \\
\end{array}$ & 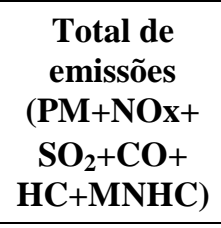 & $\begin{array}{c}\text { Água } \\
\text { consumida } \\
\text { pelos } \\
\text { funcionários }\end{array}$ \\
\hline Medidas & {$[\mathbf{C T N}]$} & {$\left[\mathbf{M J x 1 0}{ }^{3}\right]$} & {$\left[\mathbf{M J x 1 0} \mathbf{1}^{3}\right]$} & {$\left[1 \times 10^{3}\right]$} & {$[\mathbf{t}]$} & {$[\mathbf{t}]$} & {$\left[1 \times 10^{3}\right]$} \\
\hline Libra Rio & 180.529 & 41.604 & 25.999 & 10.187 & 4.728 & 54,24 & 21.611 \\
\hline Multirio & 180.344 & 44.206 & 33.013 & 10.602 & 35.668 & 353,76 & 22.493 \\
\hline $\begin{array}{l}\text { Sepetiba } \\
\text { Tecon }\end{array}$ & 225.255 & 54.381 & 33.622 & 9.431 & 6.450 & 77,42 & 20.007 \\
\hline Vila Velha & 211.387 & 37.047 & 21.666 & 7.257 & 4.139 & 53,21 & 15.396 \\
\hline Teconvi & 184.213 & 44.393 & 33.238 & 6.048 & 6.687 & 82,41 & 12.830 \\
\hline Pesos & $100 \%$ & $16,67 \%$ & $16,67 \%$ & $16,67 \%$ & $16,67 \%$ & $16,67 \%$ & $16,67 \%$ \\
\hline
\end{tabular}

${ }^{3}$ Fonte: Elaboração própria a partir de Guimarães e Leal Jr, 2013

${ }^{4}$ Fonte: Elaboração própria a partir de Guimarães e Leal Jr, 2013 


\subsection{Passo 4 -Desempenho padrão}

O quarto passo consiste em determinar os níveis de desempenho dos portos analisados. $\mathrm{O}$ procedimento de normalização foi adotado e poderá ser visto na etapa 6 e em função disso, os níveis de desempenho serão determinados como o padrão a ser adotado o que obtiver o maior resultado para o aspecto valor de serviço e o menor resultado para o aspecto influência ambiental. Os portos utilizados para efeito de comparação com o porto de Sepetiba Tecon foram selecionados a partir do numero de TEUs movimentados no ano, ficando dentro dos limites mínimo de 180.000 e máximo de 230.000 (Figura 2). Os portos selecionados e suas características, tais como quantitativo de movimentação de contêineres e quantidade de equipamentos podem ser vistos na Tabela 4.

Tabela 4 - Quantitativo de equipamentos dos portos utilizados na análise

Quantidade de Equipamentos

\begin{tabular}{|c|c|c|c|c|c|c|c|c|c|}
\hline \multirow[b]{2}{*}{ Porto } & \multirow[b]{2}{*}{ Local } & \multirow[b]{2}{*}{$\begin{array}{l}\text { Movimentação } \\
\text { (TEU's) }\end{array}$} & \multicolumn{7}{|c|}{ Quantidade de Equipamentos } \\
\hline & & & 㐫 & $\stackrel{\cup}{\underset{\Sigma}{\Sigma}}$ & 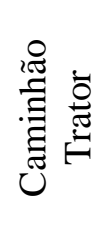 & 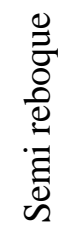 & 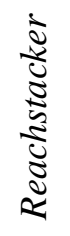 & 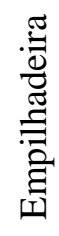 & 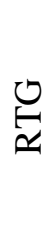 \\
\hline Libra Rio & $\begin{array}{l}\text { Rio de } \\
\text { Janeiro }\end{array}$ & 180.529 & 3 & 1 & 24 & 24 & 11 & 19 & 0 \\
\hline Multirio & $\begin{array}{l}\text { Rio de } \\
\text { Janeiro }\end{array}$ & 180.344 & 2 & 3 & 20 & 20 & 14 & 19 & 0 \\
\hline $\begin{array}{l}\text { Sepetiba } \\
\text { Tecon }\end{array}$ & Itaguaí & 225.255 & 4 & 2 & 17 & 12 & 14 & 27 & 2 \\
\hline $\begin{array}{l}\text { Vila } \\
\text { Velha }\end{array}$ & Vitória & 211.387 & 3 & 2 & 12 & 12 & 7 & 15 & 3 \\
\hline Teconvi & Itajaí & 184.213 & 2 & 4 & 0 & 0 & 16 & 0 & 0 \\
\hline
\end{tabular}

\subsection{Passo 5 - Coleta de Dados}

Neste passo, para determinar os valores dos indicadores que serão utilizados para gerar cada medida os dados são coletados. Para tal, utilizou-se como base Guimarães e Leal Jr (2013). 


\subsection{Passo 6 - Processamento}

No sexto passo, seguindo as equações de 1 a 7 , determina-se os desempenho de cada porto através dos valores encontrados para cada medida. Neste momento quanto maior o resultado para o aspecto valor de serviço e quanto menor resultado para o aspecto influência ambiental melhor será o seu desempenho. Feito isso, aplica-se a normalização das medidas e a sua agregação conforme as equações 7 e 8 respectivamente, para associar um valor único de desempenho para todos os portos analisados (Tabela 5 e 6 ).

Tabela 5 - Normalização dos Dados ${ }^{5}$

\begin{tabular}{|c|c|c|c|c|c|c|c|}
\hline \multirow{2}{*}{$\begin{array}{l}\text { Aspectos } \\
\text { Atributos }\end{array}$} & \multirow{2}{*}{$\begin{array}{c}\text { Valor de } \\
\text { Serviço } \\
\\
\text { Serviço } \\
\text { Produzido }\end{array}$} & \multicolumn{6}{|c|}{ Influência Ambiental } \\
\hline & & Consumo & de Energia & $\begin{array}{l}\text { Poluição } \\
\text { da Água }\end{array}$ & $\begin{array}{l}\text { Gases } \\
\text { Efeito }\end{array}$ & $\begin{array}{c}\text { Poluição } \\
\text { Atmosférica }\end{array}$ & $\begin{array}{l}\text { Consumo de } \\
\text { água }\end{array}$ \\
\hline 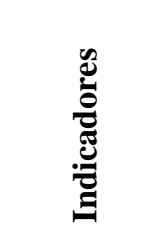 & $\begin{array}{c}\text { Numero de } \\
\text { Contêineres } \\
\text { Movimentados }\end{array}$ & $\begin{array}{c}\text { Energia } \\
\text { Total }\end{array}$ & $\begin{array}{c}\text { Energia } \\
\text { Não } \\
\text { Renovável }\end{array}$ & $\begin{array}{c}\text { Emissão } \\
\text { de Esgoto }\end{array}$ & $\begin{array}{c}\text { Total de } \\
\text { emissões } \\
\left(\mathrm{CO}_{2}\right. \\
+\mathrm{CH}_{4}+ \\
\left.\mathrm{N}_{2} \mathrm{O}\right)\end{array}$ & $\begin{array}{c}\text { Total de } \\
\text { emissões } \\
(\mathrm{PM}+\mathrm{NOx}+ \\
\mathrm{SO}_{2}+\mathrm{CO}+ \\
\mathrm{HC}+\mathrm{MNHC})\end{array}$ & $\begin{array}{c}\text { Água } \\
\text { consumida } \\
\text { pelos } \\
\text { funcionários }\end{array}$ \\
\hline Medidas & [CTN] & [MJx103] & [MJx10³] & {$\left[1 \times 10^{3}\right]$} & {$[\mathbf{t}]$} & {$[\mathbf{t}]$} & {$\left[1 \times 10^{3}\right]$} \\
\hline Libra Rio & 0,80 & 0,89 & 0,83 & 0,59 & 0,88 & 0,98 & 0,59 \\
\hline Multirio & 0,80 & 0,84 & 0,66 & 0,57 & 0,12 & 0,15 & 0,57 \\
\hline $\begin{array}{l}\text { Sepetiba } \\
\text { Tecon }\end{array}$ & 1,00 & 0,68 & 0,64 & 0,64 & 0,64 & 0,69 & 0,64 \\
\hline Vila Velha & 0,94 & 1,00 & 1,00 & 0,83 & 1,00 & 1,00 & 0,83 \\
\hline Teconvi & 0,82 & 0,83 & 0,65 & 1,00 & 0,62 & 0,65 & 1,00 \\
\hline
\end{tabular}

\subsection{Passo 7 - Avaliação}

Neste passo, após a normalização dos dados, conforme Equação 7, é possível ponderá-los e agregá-los, conforme Equação 8. Por meio da comparação dos resultados ponderados e agregados para cada alternativa é possível hierarquizá-las, destacando o porto a ser apontado como o padrão de comparação para este trabalho (Tabela 6).

\footnotetext{
${ }^{5}$ Fonte: Elaboração própria a partir de Guimarães e Leal Jr, 2013.
} 
Tabela 6 - Ponderação dos Dados

\begin{tabular}{|c|c|c|c|c|c|c|c|c|}
\hline \multirow{2}{*}{$\begin{array}{l}\text { Aspectos } \\
\text { Atributos }\end{array}$} & \multirow{2}{*}{$\begin{array}{c}\begin{array}{c}\text { Valor de } \\
\text { Serviço }\end{array} \\
\begin{array}{c}\text { Serviço } \\
\text { Produzido }\end{array}\end{array}$} & \multicolumn{6}{|c|}{ Influência Ambiental } & \\
\hline & & Consume & de Energia & $\begin{array}{l}\text { Poluição } \\
\text { da Agua } \\
\text { do Solo }\end{array}$ & $\begin{array}{l}\text { Gases } \\
\text { Efeito } \\
\text { Estufa }\end{array}$ & $\begin{array}{c}\text { Poluição } \\
\text { Atmosférica }\end{array}$ & $\begin{array}{c}\text { Consumo de } \\
\text { água }\end{array}$ & \\
\hline 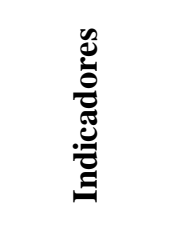 & $\begin{array}{c}\text { Numero de } \\
\text { Contêineres } \\
\text { Movimentados }\end{array}$ & $\begin{array}{c}\text { Energia } \\
\text { Total }\end{array}$ & $\begin{array}{c}\text { Energia } \\
\text { Não } \\
\text { Renovável }\end{array}$ & $\begin{array}{c}\text { Emissão } \\
\text { de Esgoto }\end{array}$ & $\begin{array}{c}\text { Total de } \\
\text { emissões } \\
\left(\mathrm{CO}_{2}\right. \\
+\mathrm{CH}_{4}+ \\
\left.\mathrm{N}_{2} \mathrm{O}\right)\end{array}$ & $\begin{array}{c}\text { Total de } \\
\text { emissões } \\
(\mathbf{P M}+\mathrm{NOx}+\mathrm{S} \\
\mathrm{O}_{2}+\mathrm{CO}+\mathrm{HC} \\
+\mathrm{MNHC})\end{array}$ & $\begin{array}{c}\text { Água } \\
\text { consumida } \\
\text { pelos } \\
\text { funcionários }\end{array}$ & Total \\
\hline Medidas & {$[\mathbf{C T N}]$} & $\begin{array}{c}\mathbf{M J x 1 0} \\
3] \\
\end{array}$ & {$\left[\mathbf{M J x 1 0}{ }^{3}\right]$} & {$\left[1 \times 10^{3}\right]$} & [t] & {$[\mathbf{t}]$} & {$\left[1 \times 10^{3}\right]$} & \\
\hline Libra Rio & 0,80 & 0,15 & 0,14 & 0,10 & 0,15 & 0,16 & 0,10 & 0,80 \\
\hline Multirio & 0,80 & 0,14 & 0,11 & 0,10 & 0,02 & 0,03 & 0,10 & 0,64 \\
\hline $\begin{array}{l}\text { Sepetiba } \\
\text { Tecon }\end{array}$ & 1,00 & 0,11 & 0,11 & 0,11 & 0,11 & 0,11 & 0,11 & 0,83 \\
\hline Vila Velha & 0,94 & 0,17 & 0,17 & 0,14 & 0,17 & 0,17 & 0,14 & 0,94 \\
\hline Teconvi & 0,82 & 0,14 & 0,11 & 0,17 & 0,10 & 0,11 & 0,17 & 0,80 \\
\hline esos & $50 \%$ & & & & $\mathbf{5 0 \%}$ & & & \\
\hline
\end{tabular}

Neste momento, quanto mais próximo de 1 (um) o resultado encontrado, melhor será o ranking dos portos e também o desempenho global podem ser verificados na Figura 2.

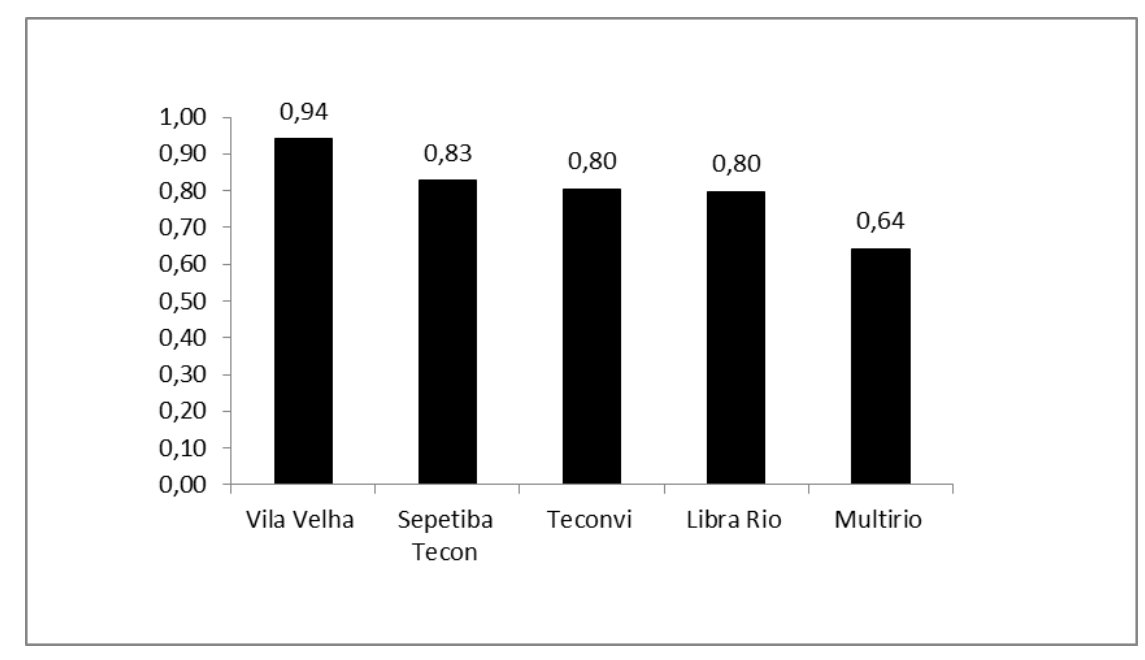

Figura 2 - Ranking do desempenho geral dos portos 


\subsection{Passo 8-Melhoria}

Por tratar-se de um passo não obrigatório do método, devido à limitação dos dados deste artigo, não foi possível a elaboração de uma proposta de melhoria, nível 2 do passo 8 , foi realizado apenas o teste de sensibilidade.

Por foi fim foi realizado um teste de sensibilidade variando-se os pesos dos aspectos, conforme Equação 9, afim de se determinar o nível de desempenho global dos portos considerando diferentes combinações de pesos (Figura 3).

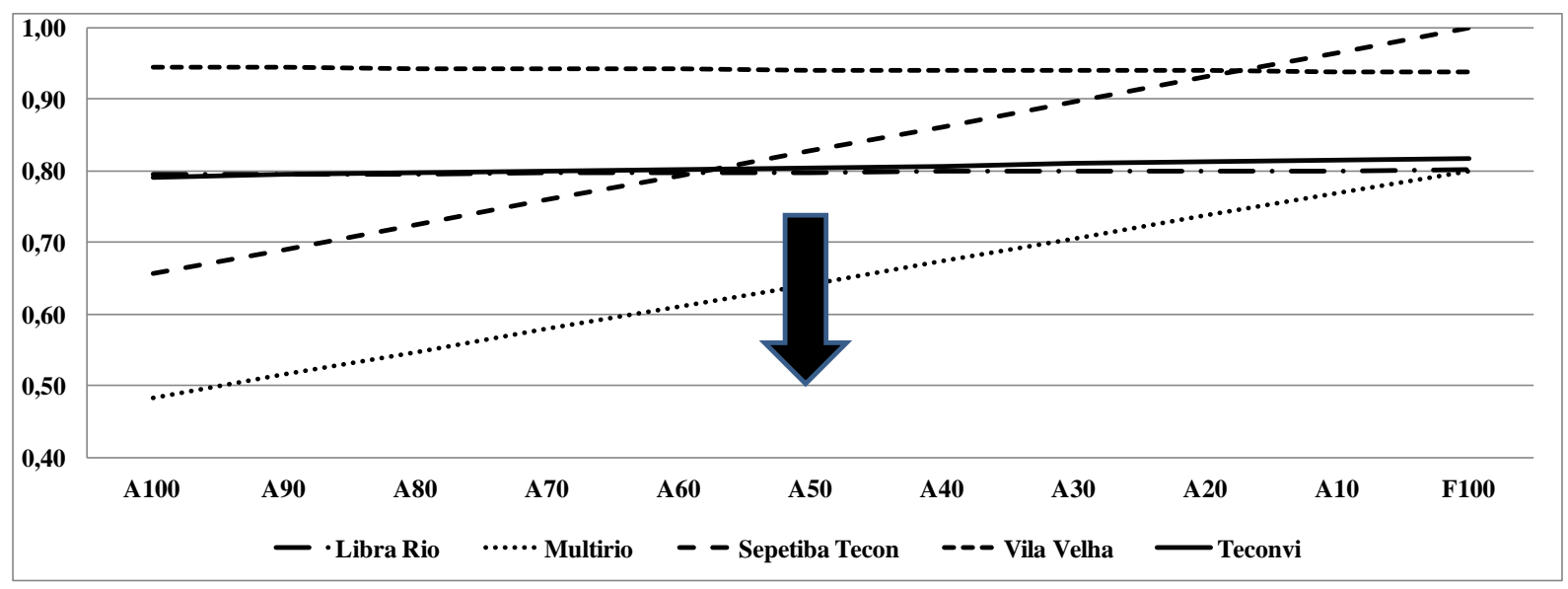

Figura 3 - Teste de Sensibilidade

Após a normalização e a ponderação dos dados e ao aplicar pesos iguais aos aspectos econômicos e ambientais, o porto de Vila Velha, com 0,94, pode ser considerado, sob a ótica global, o de melhor desempenho, sendo seu resultado $12 \%$ melhor que o porto de Sepetiba Tecon, $15 \%$ melhor que o porto de Teconvi e Libra, simultaneamente e $32 \%$ se comparado ao de pior desempenho que foi do porto Multirio. Esse resultado é decorrente do fato do porto de Vila Velha ter apresentado o melhor resultado em 5, das 8 medidas apresentadas, excetuandose apenas nas medidas de número de contêineres movimentados, emissão de esgoto e água consumida pelos funcionários, ficando nas três situações em segunda colocação (Tabela 6). 
A situação com pesos iguais a cada aspecto 50/50, já apresentada na Figura 2, pode ser verificada no meio da escala na Figura 3, realizando o teste de sensibilidade, suposições são aplicadas aos pesos de cada aspecto analisado.

Sob o ponto de vista exclusivamente ambiental, pode-se verificar que o porto Vila Velha mantém a primeira posição em relação ao desempenho, o porto que mais se aproxima dele, é o Libra Rio e o Teconvi com uma diferença de apenas 16\%, o porto de Sepetiba o porto Multirio se distanciam da primeira posição com valores que variam entre $31 \%$ e $49 \%$, respectivamente.

Já, sob o ponto de vista exclusivamente econômico, o porto Sepetiba Tecon, que foi o porto selecionado para este estudo, assume a primeira posição, se distanciando $6 \%$ do porto de Vila Velha, $18 \%$ do porto Teconvi, e $20 \%$ do portos Multirio e Libra Rio. O resultado da medida relacionada ao numero de contêineres foi decisiva para colocar o porto de Sepetiba Tecon nesta posição.

Para que o porto Sepetiba Tecon assuma a primeira colocação em ambos os aspectos estudados, é preciso que algumas ações, relacionadas às influências ambientais, sejam adotadas, tais como:

1. Redução de $8 \%$ na emissão de esgoto e água consumida por funcionário;

2. Uso de biodiesel (B100) no lugar de diesel.

3. Sistemas propulsão elétricas para guindastes, locomotivas e outros veículos.

Os atributos de avaliação, indicadores, bem como os resultados de desempenho dos portos após as adoções das medidas sugeridas acima, podem ser verificados na Tabela 7 . 
Tabela 7 - Atributos de avaliação, indicadores e medidas e desempenho dos portos

\begin{tabular}{|c|c|c|c|c|c|c|c|}
\hline \multirow{2}{*}{$\begin{array}{l}\text { Aspectos } \\
\text { Atributos }\end{array}$} & \multirow{2}{*}{$\begin{array}{c}\begin{array}{c}\text { Valor de } \\
\text { Serviço }\end{array} \\
\begin{array}{c}\text { Serviço } \\
\text { Produzido }\end{array}\end{array}$} & \multicolumn{6}{|c|}{ Influência Ambiental } \\
\hline & & Consumo & de Energia & $\begin{array}{c}\text { Poluição } \\
\text { da Âgua } \\
\text { do Solo }\end{array}$ & $\begin{array}{l}\text { Gases } \\
\text { Efeito } \\
\text { Estufa }\end{array}$ & $\begin{array}{c}\text { Poluiçãa } \\
\text { Atmosférica }\end{array}$ & $\begin{array}{c}\text { Consumo de } \\
\text { água }\end{array}$ \\
\hline Indicadores & $\begin{array}{c}\text { Numero de } \\
\text { Contêineres } \\
\text { Movimentados }\end{array}$ & $\begin{array}{c}\text { Energia } \\
\text { Total }\end{array}$ & $\begin{array}{c}\text { Energia } \\
\text { Não } \\
\text { Renovável }\end{array}$ & $\begin{array}{c}\text { Emissão } \\
\text { de } \\
\text { Esgoto }\end{array}$ & $\begin{array}{c}\text { Total de } \\
\text { emissões } \\
\left(\mathrm{CO}_{2}\right. \\
+\mathrm{CH}_{4}+ \\
\left.\mathrm{N}_{2} \mathrm{O}\right)\end{array}$ & $\begin{array}{c}\text { Total de } \\
\text { emissões } \\
(\mathbf{P M + N O x + S} \\
\text { O }_{2}+\mathrm{CO}+\mathrm{HC} \\
+\mathrm{MNHC})\end{array}$ & $\begin{array}{c}\text { Água } \\
\text { consumida } \\
\text { pelos } \\
\text { funcionários }\end{array}$ \\
\hline Medidas & [CTN] & {$\left[\mathrm{MJx}^{\mathrm{N}} \mathbf{0}^{3}\right]$} & {$\left[\mathbf{M J x} 10^{3}\right]$} & {$\left[1 \times 10^{3}\right]$} & {$[\mathbf{t}]$} & {$[\mathbf{t}]$} & {$\left[1 \times 10^{3}\right]$} \\
\hline Libra Rio & 180.529 & 41.604 & 25.999 & 10.187 & 4.728 & 54,24 & 21.611 \\
\hline Multirio & 180.344 & 44.206 & 33.013 & 10.602 & 35.668 & 353,76 & 22.493 \\
\hline $\begin{array}{l}\text { Sepetiba } \\
\text { Tecon }\end{array}$ & 225.255 & 54.381 & 0,0 & 8.705 & 1.372 & 64,55 & 18.467 \\
\hline Vila Velha & 211.387 & 37.047 & 21.666 & 7.257 & 4.139 & 53,21 & 15.396 \\
\hline Teconvi & 184.213 & 44.393 & 33.238 & 6.048 & 6.687 & 82,41 & 12.830 \\
\hline Pesos & $100 \%$ & $16,67 \%$ & $16,67 \%$ & $16,67 \%$ & $16,67 \%$ & $16,67 \%$ & $16,67 \%$ \\
\hline
\end{tabular}

Além disso, o ranking total dos portos após a adoção das medidas de melhoria está apresentado na Figura 4.

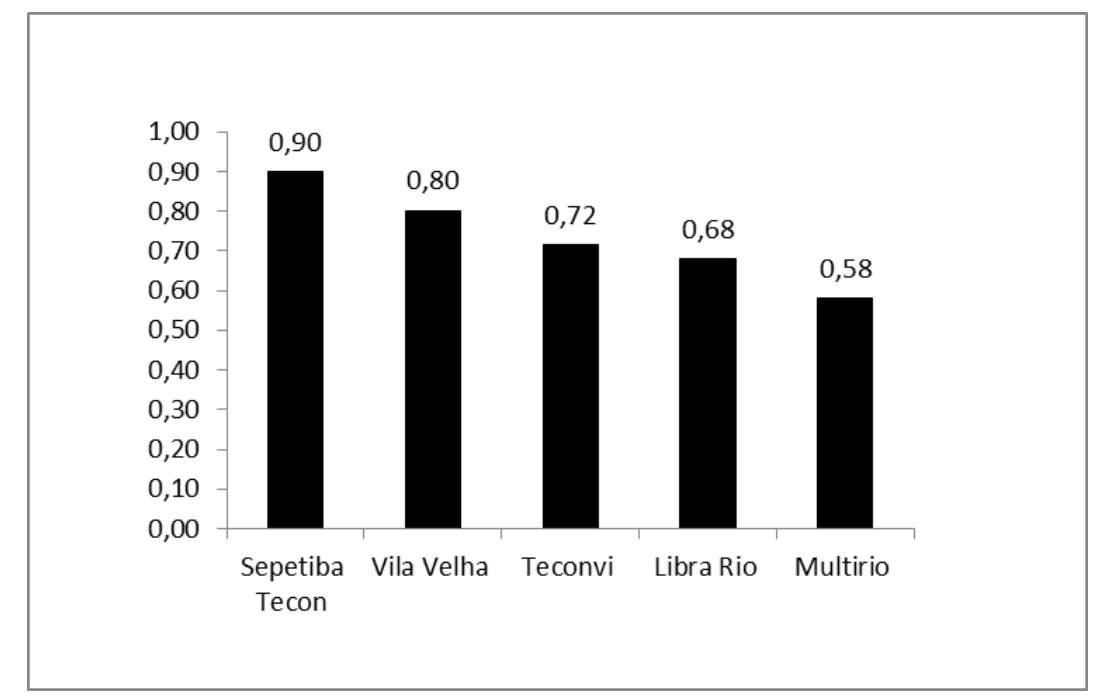

Figura 4 - Ranking total após adoção de medidas de melhoria 
Os novos valores foram encontrados através da adoção das medidas recomendadas. Para a redução da emissão de esgoto e água consumida, o valor de $8 \%$ foi recomendado com base no consumo médio dos portos analisados, pois se verificou que ele estava acima da média. Esse resultado pode ser alcançado através de medidas de conscientização dos colaboradores. Para a diminuição da utilização de energia não renovável, considerou-se o uso de Biodiesel (B100) e adoção de equipamentos elétricos no lugar de equipamentos de fontes não renováveis, passando assim, a consumir somente energia renovável e para esta medida, assumindo o valor igual a 0 . Esta medida pode ser adotada, especialmente no Brasil, devido à energia elétrica ser de origem renovável. Para a redução do $\mathrm{MP}, \mathrm{SO}^{2}, \mathrm{CO}$ e $\mathrm{CO}^{2}$, baseu-se em Leal Jr (2010), conforme Tabela 8.

Tabela 8 - Impactos na emissão de poluentes pela utilização do B100

\begin{tabular}{lccccc}
\multicolumn{1}{c}{ Fonte } & NOx & MP & SOx & CO & CO $^{2}$ \\
\hline Oliveira e Costa (2001) & $13,00 \%$ & $-50,00 \%$ & $-98 \%$ & - & $78,00 \%$ \\
Silva (2007) & - & $-32,00 \%$ & $-100 \%$ & - & $78,50 \%$ \\
USDA e USDOE (1998) & $8,90 \%$ & $-68,10 \%$ & $-100 \%$ & $-46,20 \%$ & $78,50 \%$ \\
Pacific Biodiesel (2000) & $10,00 \%$ & $-68,00 \%$ & - & $-67,00 \%$ & - \\
EPA (2002) & $10,00 \%$ & $-47,00 \%$ & $-100 \%$ & $-48,00 \%$ & - \\
\hline Média utilizada & $\mathbf{1 0 , 5 0 \%}$ & $\mathbf{- 5 3 , 0 0 \%}$ & $\mathbf{- 9 9 , 5 0 \%}$ & $\mathbf{- 5 3 , 7 0 \%}$ & $\mathbf{- 7 8 , 3 0 \%}$ \\
\hline
\end{tabular}

\section{Conclusão}

Através da aplicação do Método de avaliação de desempenho ambiental de PGVs carga (Guimarães et al. 2013) foi possível avaliar o desempenho de quatro portos, com o numero de TEUs movimentados no período de um ano, dentro dos limites mínimo de 180.000 e máximo de 230.000 e comparar o desempenho com o do porto de Sepetiba Tecon.

Verificou-se que, sob o ponto de vista exclusivamente econômico o porto de Sepetiba Tecon apresenta um melhor desempenho em relação aos demais, no entanto, sob o ponto de vista exclusivamente ambiental, apresenta-se em quarto lugar, variando cerca de $52 \%$ entre um extremo e outro. Para os resultados encontrados nesta aplicação é preciso considerar a limitação em relação à aquisição dos dados, já que estes foram adquiridos a partir de fontes secundárias e também não foi encontrado na bibliografia utilizada, valores para redução de $\mathrm{N}_{2} \mathrm{O}, \mathrm{CH}_{4}$ e $\mathrm{HC}+\mathrm{MNHC}$. 
Como proposta para novos estudos sugere-se a avaliação dos indicadores considerando-se dados numéricos. Desta forma a análise poderá ser realizada com base no princípio de proporcionalidade, ou seja, diferentes pesos para diferentes quantitativos de movimentação de contêineres.

Além disso, sugere-se como potencial estudo futuro a análise não apenas da operação dentro dos terminais, mas também das viagens geradas pelo terminal, considerando em especial os impactos ambientais gerados pelas viagens (veículos de carga) que são atraídas pelo terminal. Outro aspecto que poderia ser contabilizado na análise é o impacto das viagens motorizadas dos funcionários.

\section{Referências}

Associação Brasileira dos Terminais de Contêineres de Uso Público - ABRATEC (2013) Estatísticas.

Companhia de Engenharia de Tráfego - CET (1983) Polos Geradores de Tráfego. Boletim Técnico n. 32. São Paulo.

Companhia Siderúrgica Nacional - CSN (2008) Plataforma logística CNS em Itaguaí. Um projeto alinhado com as necessidades do Brasil. Apresentação institucional (não publicado).

Confederação Nacional dos Transportes - CNT (2012) Pesquisa CNT do Transporte Marítimo 2012. Porto de Itaguaí - RJ. Apresentação institucional (não publicado).

Departamento Nacional de Trânsito - DENATRAN (2001) Manual de procedimentos para o tratamento de polos geradores de tráfego. Ministério da Justiça. Brasília.

D’Agosto, M. A, Souza, C. D. R, Campos, V. B. G. (2012) Modelos de geração de viagem para polos geradores de viagens de carga. In: Polos geradores de viagem orientados a qualidade de vida e ambiental. Org: Licínio da Silva Portugal. Rio de Janeiro: Editora Interciência.

Guimarães, V. A. e Leal Jr, I. C. (2013) Análise da ecoeficiência em terminais de contêineres brasileiros. XVI Congreso Chileno de Ingeniería en Transport, Santiago.

Guimarães. V. A, Leal Jr. I. C, D’Agosto, M. A. Oliveira, C. M, e Ferreira. A. F. (2013) Desempenho ambiental de polos geradores de viagem de carga. Journal of Tranport Literature, vol. 8, n. 3.

D’Agosto, M.A, Souza, C. D. R, Silva, S. D, Silva, M. A. V, Leal Jr, I.C, Rodrigues, J. M. M, Almeida, M. L. R e Barboza, A.P. (2011) Inventário de Emissões Atmosféricas por Veículos Automotores do Estado do Rio de Janeiro. Relatório final (não publicado).

Kneib, E. C. Silva, P. C. M. e Portugal, L. S. (2010) Impactos decorrentes da implantação de pólos geradores de viagens na estrutura espacial das cidades. Transportes, vol. 18, n. 1, p. 27-35.

Leal Jr. I. C, Mendes, M. A. S, Guimarães, V. A. e Ribeiro, A. A. M. (2012) Avaliação da ecoeficiência nas operações portuárias com a utilização da análise relacional Grey. XXVI ANPET, Joinville.

Leal Jr, I.C, D’Agosto, M. A. (2011) Modal choice for transportation of hazardous materials: the case of land modes of transport of bio-ethanol in Brazil. Journal of Cleaner Production, vol. 19, pp. 
229-240.

Leal Jr, I. C. (2010) Método de Escolha Modal para transporte de produtos perigosos com base em medidas de ecoeficiência. Tese de doutorado. UFRJ/COPPE. Rio de Janeiro.

Starcrest Consulting Group - LLC (2011a) Port of Los Angeles: Inventory of Air Emission 2010.

Starcrest Consulting Group - LLC (2011b) Air Emissions Inventory 2010: Port of Long Beach Centennial Celebration.

Starcrest Consulting Group - LLC (2012) Air Emissions Inventory 2011: Port of Long Beach, the Green Port.

Portugal, L. S. e Goldner, L. G. (2003) Estudo de Pólos Geradores de Tráfego e de seus Impactos nos Sistemas Viários e de Transportes. Rio de Janeiro: Editora Edgard Blucher.

Rede PGV (2013) Portal da Rede Ibero-americana de Estudo em Pólos Geradores de Viagem. Disponível em redpgv.coppe.ufrj.br.

Souza, C. D. R., Silva, S. D., D’Agosto, M. A. (2010) Modelos de geração de viagem para pólos geradores de viagens de cargas. Transportes, vol. 18, n. 1, pp. 46-57.

Tolfo, J. D e Portugal, L. S. (2006) Uso de micro-simulador na análise de desempenho viário em redes com pólos geradores de viagens. $2^{\circ}$ PLURIS - Congresso Luso Brasileiro para o Planejamento, Urbano, Regional, Integrado, Sustentável. Braga. 